\title{
Optimum User Selection for Hybrid-Duplex Device-to-Device in Cellular Networks
}

\author{
Gaojie Chen, Dandan Liang, Mir Ghoraishi, Pei Xiao and Rahim Tafazolli \\ Institute for Communication Systems (ICS), home of $5 G$ Innovation Centre \\ Department of Electronic Engineering, Faculty of Engineering and Physical Sciences \\ University of Surrey, Guildford, Surrey, UK \\ Email:\{gaojie.chen, d.liang, m.ghoraishi, p.xiao and r.tafazolli\}@surrey.ac.uk
}

\begin{abstract}
This paper studies the optimum user selection scheme in a hybrid-duplex device-to-device (D2D) cellular networks. We derive an analytical integral-form expression of the cumulative distribution function (CDF) for the received signalto-noise-plus-interference-ratio (SINR) at the D2D node, based on which the closed-form of the outage probability is obtained. Analysis shows that the proposed user selection scheme achieves the best SINR at the D2D node with interference to base station being limited by a pre-defined level. Hybrid duplex D2D can be switched between half and full duplex according to different residual self-interference to enhance the throughput of D2D pair. Simulation results are presented to validate the analysis.

Index Terms -Device-to-Device (D2D) communications, uplink cellular networks, throughput, cellular user selection.
\end{abstract}

\section{INTRODUCTION}

Device-to-device (D2D) communications has been considered as a key technique in 5 th generation $(5 \mathrm{G})$ communications because of its several advantages, i.e., throughput enhancement, user equipment power savings, and instantaneous data rate increase [1]. For D2D underlay cellular networks, D2D users send data signals to each other over a direct link by reusing cellular resources, similar to the secondary user scenario introduced in cognitive radio systems [2]. However, the major differences between the D2D and cognitive radio systems is the radio resource usage can be controlled in cooperation with cellular networks in the D2D underlay scenario, whereas the secondary user in cognitive radio systems is not controlled by the primary user networks [3].

Furthermore, current wireless communication networks usually employ half-duplex model. Most existing work has been targeted at half duplex device-to-device (HD-D2D) networks including resource allocation [4], and power optimization [5]. However half-duplex transmission incurs $50 \%$ loss in spectral efficiency. In order to improve spectral efficiency, full duplex transmission, which was previously considered impractical due to the associated self-interference (SI), has been considered recently because of the advances in SI cancelation approaches 978-1-4673-6540-6/15/\$31.00 (c)2015 IEEE (e.g. [6], [7]). Furthermore, [8] and [9] have combined the concepts of full duplex and D2D to improve the system sumrate, because it can harvest the advantages of both technologies to improve the spectral efficiency of wireless communications. Most previous works implied Doppler's mechanism [3] in which the base station (BS) controls the maximum transmit power of the D2D transmitter which can efficiently manage the D2D interference to BS in upper link. However the interference from cellular user to D2D receiver has not been considered in aforementioned works.

In this paper, we consider cellular user selection scheme in hybrid-duplex D2D cellular networks with uplink underlaying policy, where one BS, one D2D pair and $K$ available cellular users. We not only consider efficient interference coordination to prevent harmful interference to $\mathrm{BS}$, but also achieve throughput enhancement of D2D systems by proper design of the cellular user selection scheme. We can also switch the hybrid-duplex between half and full duplex according to residual SI to improve the throughput of D2D link. Moreover, satisfying these conditions makes it very hard to obtain the distribution of the SINR and the related outage probability at the D2D node. The main contribution of this paper, therefore, is to derive an integral-form expression of the outage probability for the cellular user selection in a hybrid-duplex D2D cellular networks. The analysis not only provides a deep insight into understanding cellular user selection in an interference limited D2D cellular system, but also suggestion an interesting way for analyzing similar systems.

The remainder of this paper is organized as follows. The system model is presented in Section II. In Section III and IV, the cellular user selection in half and full duplex D2D networks are analyzed, respectively. The throughput of D2D networks analysis verification and conclusions are provided in Section V and Section VI, respectively.

Notations: we use upper bold-face letters to represent matrices and vectors. $\operatorname{Ei}(1, \mathrm{a})$ denotes an exponential integral. And $f($.$) and F($.$) denote the PDF and CDF, respectively.$ 


\section{System Model}

A cellular network with hybrid-duplex D2D is shown in Fig. 1, where there is one cellular base station and $K$ available users ${ }^{1}$ from $S_{1}$ to $S_{K}$ and a hybrid-duplex D2D pair. We assume the BS and each user are half-duplex devices and D2D pair can switch between half-duplex and full-duplex modes. The channels $S i \rightarrow B S, D_{1} \rightarrow B S$, $D_{2} \rightarrow B S, S_{i} \rightarrow D_{1}, S_{i} \rightarrow D_{2}, D_{1} \rightarrow D_{1}$ and $D_{2} \rightarrow D_{2}$, which are denoted as $h_{s_{i} b}, h_{d_{1} b}, h_{d_{2} b}, h_{s_{i} d_{1}}, h_{s_{i} d_{2}}, h_{d_{1} d_{1}}$ and $h_{d_{2} d_{2}}$, respectively, are independently Rayleigh flat fading, and keep unchanged within one packet but may vary from one packet to another have been assumed. Therefore, the corresponding channel gains, obtained as $\gamma_{j}=\left|h_{j}\right|^{2}\left(j \in\left\{s_{i} b, d_{1} b, d_{2} b, s_{i} d_{1}, s_{i} d_{2}, d_{1} d_{1}, d_{2} d_{2}\right\}\right)$ respectively, are independently exponentially distributed with mean of $\lambda_{j}$, respectively. We assume the channel information between each user and full-duplex device-to-device (FD-D2D) pair can be obtained by the $\mathrm{BS}^{2}$. In the cellular cell, the $K$ available users attempt to transmit signals to the base station BS by using uplink resource. In order to enhance the throughput of D2D link, the best user will be selected by BS and share same uplink resources (i.e., time slot and frequency bin) with current D2D user.

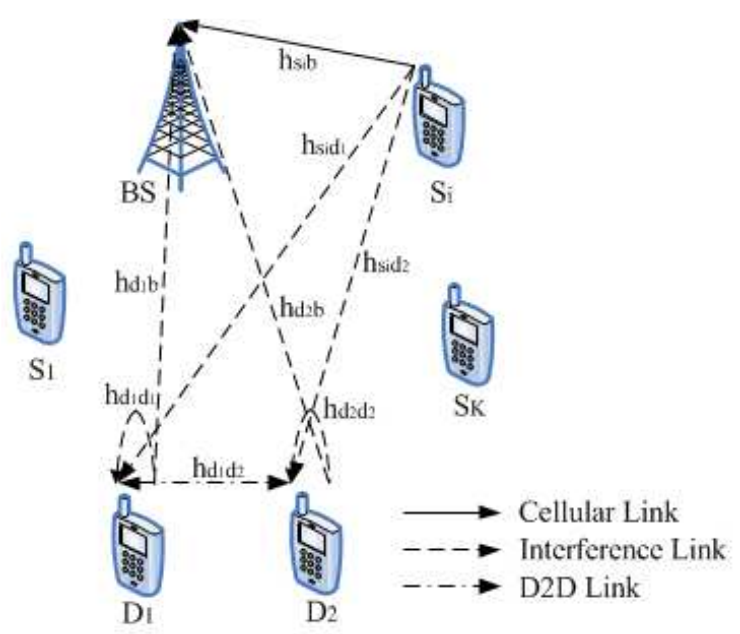

Fig. 1. A cellular network with a hybrid-duplex D2D pair and $K$ available cellular users with three different links.

In our proposed system, the maximum power of D2D transmitter is strictly limited so as not to generate any harmful

\footnotetext{
${ }^{1}$ The available users means the users that can be guaranteed a reliable communicate with BS

${ }^{2}$ The CSI is usually estimated through pilots and feedback (e.g. [10]), and the CSI estimation without feedback may also be applied (e.g [11]), which are beyond the scope of this paper.
}

interference to BS, and can be defined

$$
P_{d_{1}} \leq \min \left(\frac{I_{t h}}{\gamma_{d_{1} b}}, \bar{P}\right) \quad \text { and } \quad P_{d_{2}} \leq \min \left(\frac{I_{t h}}{\gamma_{d_{2} b}}, \bar{P}\right)
$$

where $I_{t h}$ is the interference threshold, and $\bar{P}$ is the maximum transmission power as in [6]. All noises are additive white Gaussian noise. Without losing generality, the noise variances and transmission power $P_{s}$ for the each cellular user are all normalized to unity. In the next section, we will analyze the cellular user selection for half duplex D2D transmission.

\section{Cellular USer Selection in Half Duplex D2D NETWORKS}

For half duplex D2D system, $D_{1}$ transmits signal to $D_{2}$ sharing uplink radio resource with the selected user. Therefore, the received signal according to different time slots at the $D_{2}$ is given by

$$
\mathbf{y}_{d_{2}, i}^{H}=\sqrt{P_{d_{1}}} \mathbf{x}_{d_{1}} h_{d_{1} d_{2}}+\sqrt{P_{s}} \mathbf{x}_{s} h_{s_{i} d_{2}}+\mathbf{n}_{d_{2}},
$$

where $\mathbf{x}_{d_{1}}$ and $\mathbf{x}_{s}$ are transmission vectors from $D_{1}$ and $S_{i}$ respectively, and $\mathbf{n}_{d_{2}}$ is the noise vector at $D_{2}$ with zero mean and covariance matrix of $\sigma_{d_{2}}^{2} \mathbf{I}$, where $\mathbf{I}$ is an identity matrix. According to (1) and (2), at high SNRs, the instantaneous SINR from $D_{1}$ to $D_{2}$ can be obtained as

$$
\gamma_{d_{2}, i}^{H}=\frac{P_{d_{1}}\left|h_{d_{1} d_{2}}\right|^{2}}{P_{s}\left|h_{s_{i} d_{2}}\right|^{2}+\sigma_{d_{2}}^{2}} \simeq \frac{\min \left(\frac{I_{t h}}{\gamma_{d_{1} b}}, \bar{P}\right) \gamma_{d_{1} d_{2}}}{\gamma_{s_{i} d_{2}}},
$$

where $\gamma_{d_{1} d_{2}}$ and $\gamma_{s_{i} d_{2}}$ are channel gains between $D_{1}$ and $D_{2}$ and between $S_{i}$ and $D_{2}$, respectively.

In this work, the SINR of D2D link can be enhanced by the optimum cellular user selection as

$$
\gamma_{d_{2}}^{H}=\max _{i \in K}\left\{\gamma_{d_{2}, i}^{H}\right\}=\frac{\min \left(\frac{I_{t h}}{\gamma_{d_{1} b}}, \bar{P}\right) \gamma_{d_{1} d_{2}}}{\min _{i \in K}\left\{\gamma_{s_{i} d_{2}}\right\}} .
$$

Next, we first derive expressions for the probability-densityfunction (PDF) and cumulative-distribution-function (CDF) of the SINR in (4), and then obtain the outage probability for the D2D pair. For better exposition, we let $x=\gamma_{d_{1} b}$, $y=\gamma_{d_{1} d_{2}}$ and $w=\min _{i \in K}\left\{\gamma_{s_{i} d_{2}}\right\}$. The PDF of $y$ is $f_{Y}(y)=$ $1 / \lambda_{d_{1} d_{2}} e^{-y / \lambda_{d_{1} d_{2}}}$ and the CDF of $w$ can be obtained as

$$
F_{W}(w)=e^{-\frac{K}{\lambda_{s d_{2}} w}} .
$$

The CDF of $t=y w$ can thus be derived as

$$
F_{T}(t)=\int_{0}^{\infty} f_{Y}(y) F_{W}(t / y) d y=\frac{\lambda_{s d_{2}} t}{K \lambda_{d_{1} d_{2}}+\lambda_{s d_{2}} t} .
$$


Next we can obtain the CDF of $\gamma_{d_{2}}=\min \left(\frac{I_{t h}}{x}, \bar{P}\right) t$ as

$$
\begin{aligned}
& F_{\gamma_{d_{2}}}(\gamma)=\int_{I_{t h} / \bar{P}}^{\infty} \int_{0}^{\gamma x / I_{t h}} f_{X}(x) f_{T}(t) d t d x \\
& +\int_{0}^{I_{t h} / \bar{P}} \int_{0}^{\gamma / \bar{P}} f_{X}(x) f_{T}(t) d t d x \\
& =\frac{\lambda_{s d_{1}} \gamma+K \bar{P} \lambda_{d_{1} d_{2}} e^{-\frac{I_{t h}}{\bar{P} \lambda_{d 1 b}}}}{K \bar{P} \lambda_{d_{1} d_{2}}+\lambda_{s d_{2}} \gamma}-\alpha e^{\varphi} \operatorname{Ei}\left(1, \varphi+\frac{1}{\bar{P} \lambda_{d_{1} b}}\right),
\end{aligned}
$$

where $\varphi=\frac{I_{t h} K \lambda_{d_{1} d_{2}}}{\lambda_{s d_{2}} \gamma \lambda_{d_{1} b}}$ and $\operatorname{Ei}(1, a)$ is an exponential integral as $\operatorname{Ei}(1, a)=\int_{1}^{\infty} \frac{\exp (-t a)}{a} d t, a>0$. The outage event occurs when the SINR at the D2D pair falls below a certain target level. From (7), the outage probability for the proposed relay selection system is given by

$$
P_{\text {out }}^{H}=\int_{0}^{\alpha} f_{\gamma_{d_{2}}^{H}}(\gamma) d \gamma=F_{\gamma_{d_{2}}^{H}}(\alpha),
$$

where $\alpha$ is the pre-defined target SINR which is $2^{R_{T}}-1$. In this work we consider the delay-limited transmission mode, where the average throughput can be calculated by the outage probability of the system at a fixed transmission rate $R_{T}$ $\mathrm{b} / \mathrm{s} / \mathrm{Hz}$. In the HD-D2D scenario, the throughput can be calculated as

$$
T_{H}=R_{T}\left(1-P_{\text {out }}^{H}\right) .
$$

In the next section, the FD-D2D scenario will be analyzed.

\section{Cellular user selection in Full Duplex D2D NETWORKS}

For FD-D2D systems, the best cellular user will be selected from $K$ available users to share the same uplink radio resources with a FD-D2D pair, and $D_{1}$ and $D_{2}$ transmit signals to each other enabled by SI cancellation. As we mention earlier, with the current state-of-the-art technology, the SI can be nearly cancelled to noise level [6], [7]. Therefore, the received signal according to different time slots at the $D_{2}$ is given by

$\mathbf{y}_{d_{2}, i}^{F}=\sqrt{P_{d_{1}}} \mathbf{x}_{d_{1}} h_{d_{1} d_{2}}+\sqrt{P_{d_{2}}} \mathbf{x}_{d_{2}} h_{d_{2} d_{2}}+\sqrt{P_{s}} \mathbf{x}_{s} h_{s_{i} d_{2}}+\mathbf{n}_{d_{2}}$,

where $\mathbf{x}_{d_{2}}$ is transmission vectors from $D_{2}$. According to (1) and (10), at high SNRs, the instantaneous SINR at $D_{2}$ can be obtained as

$$
\begin{aligned}
\gamma_{d_{2}, i}^{F} & =\frac{P_{d_{1}}\left|h_{d_{1} d_{2}}\right|^{2}}{P_{d_{2}}\left|h_{d_{2} d_{2}}\right|^{2}+P_{s}\left|h_{s_{i} d_{2}}\right|^{2}+\sigma_{d_{2}}^{2}} \\
& \simeq \frac{\min \left(\frac{I_{t h}}{\gamma_{d_{1} b}}, \bar{P}\right) \gamma_{d_{1} d_{2}}}{\min \left(\frac{I_{t h}}{\gamma_{d_{2} b}}, \bar{P}\right) \gamma_{d_{2} d_{2}}+\gamma_{s_{i} d_{2}}}
\end{aligned}
$$

The maximum capacity of FD-D2D link can be obtained by the optimum cellular user selection as

$$
\gamma_{d_{2}}^{F}=\max _{i \in K}\left\{\gamma_{d_{2}, i}^{F}\right\}=\frac{\min \left(\frac{I_{t h}}{\gamma_{d_{1} b}}, \bar{P}\right) \gamma_{d_{1} d_{2}}}{\min \left(\frac{I_{t h}}{\gamma_{d_{2} b}}, \bar{P}\right) \gamma_{d_{2} d_{2}}+\min _{i \in K}\left\{\gamma_{s_{i} d_{2}}\right\}} .
$$

Next, we derive expressions for the PDF and CDF of the SINR in (12), and then obtain the outage probability for the overall system. For better exposition, we let $z=\gamma_{d_{2} b}$, the PDF of $z$ is $f_{Z}(z)=1 / \lambda_{d_{2} b} e^{-z / \lambda_{d_{2} b}}$. Therefore, by using the same calculation as (7), the CDF and PDF of $A=\min \left(\frac{I_{t h}}{\gamma_{d_{1} b}}, \bar{P}\right) \gamma_{d_{1} d_{2}}$ can be derived as

$$
\begin{aligned}
F_{A}(a)= & 1-e^{-\frac{a}{\bar{P} \lambda_{d_{1} d_{2}}}}+\frac{\lambda_{d_{1} b} a}{I_{t h} \lambda_{d_{1} d_{2}}+\lambda_{d_{1} b} a} e^{-\frac{I_{t h} \lambda_{d_{1} d_{2}}+\lambda_{d_{1} b}{ }^{a}}{\bar{P} \lambda_{d_{1} d_{2} \lambda_{d_{1}} b}}}, \\
f_{A}(a)= & \frac{e^{-\frac{a}{\bar{P} \lambda_{d_{1} d_{2}}}}}{\bar{P} \lambda_{d_{1} d_{2}}}+\frac{\lambda_{d_{1} b}\left(I_{t h} \lambda_{d_{1} d_{2}}^{2} \bar{P}-\lambda_{d_{1} b} a-I_{t h} \lambda_{d_{1} d_{2}}^{2} a\right)}{\left(I_{t h} \lambda_{d_{1} d_{2}}+\lambda_{d_{1} b} a\right)^{2} \lambda_{d_{1} d_{2}} \bar{P}} \\
& e^{-\frac{I_{t h} \lambda_{d_{1} d_{2}}+\lambda_{d_{1} b} a}{\bar{P} \lambda_{d_{1} d_{2} \lambda_{d_{1} b}}}},
\end{aligned}
$$

respectively, and the CDF of $B=\min \left(\frac{I_{t h}}{\gamma_{d_{2} b}}, \bar{P}\right) \gamma_{d_{2} d_{2}}$ can be obtained as

$F_{B}(b)=1-e^{-\frac{b}{\bar{P} \lambda_{d_{2} d_{2}}}}+\frac{\lambda_{d_{2} b} b}{I_{t h} \lambda_{d_{2} d_{2}}+\lambda_{d_{2} b} b} e^{-\frac{I_{t h} \lambda_{d_{2} d_{2}}+\lambda_{d_{2} b^{b}}}{\bar{P} \lambda_{d_{2} d_{2} d_{2} b}}}$.

Then the CDF of $D=B+w$ is obtained as

$$
\begin{aligned}
& F_{D}(d)=\int_{0}^{d} \int_{0}^{d-w} f_{B}(b) f_{W}(w) d b d w \\
& =-\frac{1}{\lambda_{d_{2} b} \lambda_{s d_{2}}(K) \bar{P}}\left[I_{t h} K^{2} \bar{P} \lambda_{d_{2} d_{2}}^{2} \nu(\operatorname{Ei}(1, \theta)\right. \\
& -\operatorname{Ei}(1, \mu))-K \bar{P} \lambda_{d_{2} b} \lambda_{d_{2} d_{2}} \lambda_{s d_{2}}\left(1-e^{-\frac{d}{\bar{P} \lambda_{d_{2} d_{2}}}}\right) \\
& -I_{t h} K \bar{P} \lambda_{d_{2} d_{2}} \lambda_{s d_{2}} \nu(\operatorname{Ei}(1, \theta)-\operatorname{Ei}(1, \mu))
\end{aligned}
$$

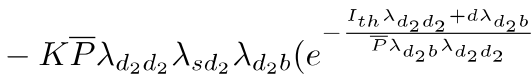

$$
\begin{aligned}
& \left.\left.-e^{-\frac{I_{t h} \lambda_{s d_{2}}+d K \bar{P} \lambda_{d_{2} b}}{\bar{P} \lambda_{d_{2} b}{ }^{\lambda} s d_{2}}}\right)+\lambda_{d_{2} b} \lambda_{s d_{2}}^{2}\left(1-e^{-\frac{K d}{\lambda_{s d_{2}}}}\right)\right] \text {, }
\end{aligned}
$$

where $\theta=-\frac{I_{t h}\left(K \bar{P} \lambda_{d_{2} d_{2}}-\lambda_{s d_{2}}\right)}{\bar{P} \lambda_{d_{2} b} \lambda_{s d_{2}}}, \quad \mu=$ $-\frac{I_{t h} K \bar{P} \lambda_{d_{2} d_{2}}^{2}+K \bar{P} d \lambda_{d_{2} b} \lambda_{d_{2} d_{2}}+I_{t h} \lambda_{s d_{2}} \lambda_{d_{2} d_{2}}-d \lambda_{s d_{2}} \lambda_{d_{2} b}}{\bar{P} \lambda_{L_{2}}} \quad$ and

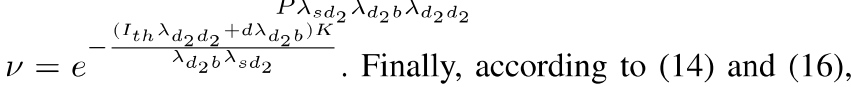
the CDF of $\gamma_{d 2}^{F}$ can be obtained as

$$
F_{\gamma_{d 2}^{F}}(\gamma)=\int_{0}^{\infty} f_{A}(a) F_{D}(a / \gamma) d a
$$


While (17) is in an integral form, it can be easily evaluated numerically with, for example Matlab or Maple [12]. We can derive the outage probability from $D_{1}$ to $D_{2}$ as $P_{\text {out }}^{F_{1}}$ by using the same method as (8). Furthermore, in the delay-limited transmission mode, the average throughput can be calculated by the outage probability of the system at a fixed transmission rate. The throughput of FD-D2D scenario can be calculated as

$$
T_{F}=R_{T}\left(1-P_{\text {out }}^{F_{1}}\right)+R_{T}\left(1-P_{\text {out }}^{F_{2}}\right),
$$

where $P_{\text {out }}^{F_{2}}$ is the outage probability from $D_{2}$ to $D_{1}$ which can be calculated by the same method with $P_{\text {out }}^{F_{1}}$. In the next section, the throughput of half and full duplex D2D will be assessed by simulations.

\section{Simulations}

In this section, simulation results are presented to verify the above analysis. In the simulations below, the noise variances $\sigma_{d_{1}}^{2}$ and $\sigma_{d_{2}}^{2}$ and each cellular user transmission power are all normalized to unity.

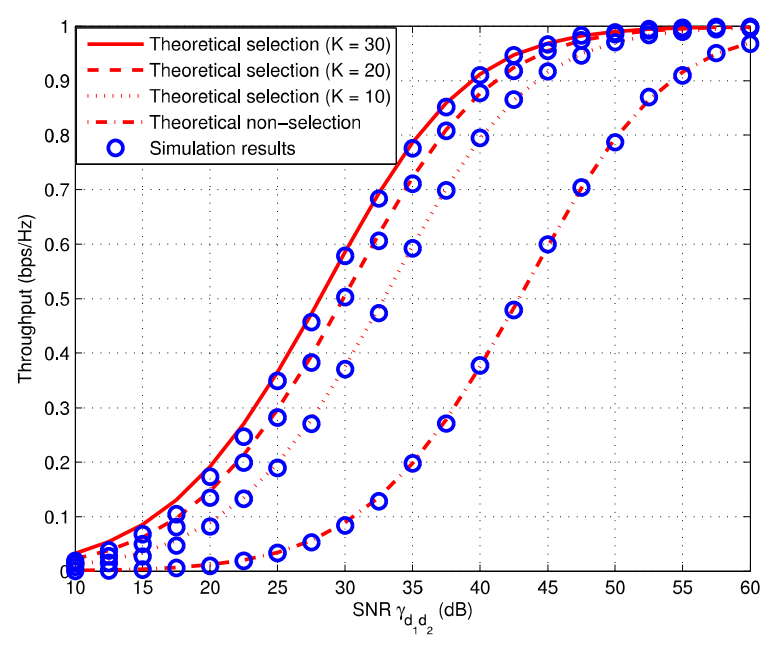

Fig. 2. Theoretical $v s$ numerical throughput of HD-D2D with different number of available users, where $I_{t h}=2, \gamma_{d_{1} b}=15 \mathrm{~dB}, \gamma_{s d_{2}}=30 \mathrm{~dB}$ and $R_{T}=$ 1 .

Fig. 2 compares the theoretical analysis with the simulation results, where $I_{t h}=2, \gamma_{d_{1} b}=15 \mathrm{~dB}, \gamma_{s d_{2}}=30 \mathrm{~dB}$ and $R_{T}$ $=1$. The analytical results based on the approximation (9) are shown, and the simulation results are obtained by averaging over 1,000,000 independent runs. The results are compared under different settings of the mean channel gain $\gamma_{d_{1} d_{2}}$ and number of available cellular users. It is obviously that under good channel conditions when the channel gains are high, the theoretical approximate (9) and simulated throughput are very well matched. It is also shown in Fig. 2 that the throughput is significantly improved by using our proposed cellular user selection scheme. For example, when $\gamma_{d_{1} d_{2}}=30 \mathrm{~dB}$, the throughput with non-selection is around $0.13 \mathrm{bps} / \mathrm{Hz}$, however, the throughput of our proposed scheme are $0.5,0.63$ and 0.7 bps $/ \mathrm{Hz}$ for $K=10,20$ and 30 , respectively.

Fig. 3 compares the theoretical analysis with the simulation results, where $I_{t h}=1, \gamma_{d_{1} b}=15 \mathrm{~dB}, \gamma_{s d_{2}}=30 \mathrm{~dB}, \gamma_{d_{1} d_{1}}=$ $\gamma_{d_{2} d_{2}}=5 \mathrm{~dB}$ and $R_{T}=1$. The analytical results based on the approximation (18) are shown, and the simulation results are also obtained by averaging over 1,000,000 independent runs. The results are compared under different settings of the mean channel gain $\gamma_{d_{1} d_{2}}$ and number of available users. It is obviously that under the good channel conditions, the theoretical approximate (18) and simulated throughput match very closely. It is also shown in Fig. 3 that the throughput is significantly improved by using our proposed user selection scheme, i.e., the throughput of non-selection is around 0.2 $\mathrm{bps} / \mathrm{Hz}$, however, the throughput of our proposed scheme are $0.72,1$ and $1.15 \mathrm{bps} / \mathrm{Hz}$ for $K=10,20$ and 30, respectively, when $\gamma_{d_{1} d_{2}}=30 \mathrm{~dB}$.

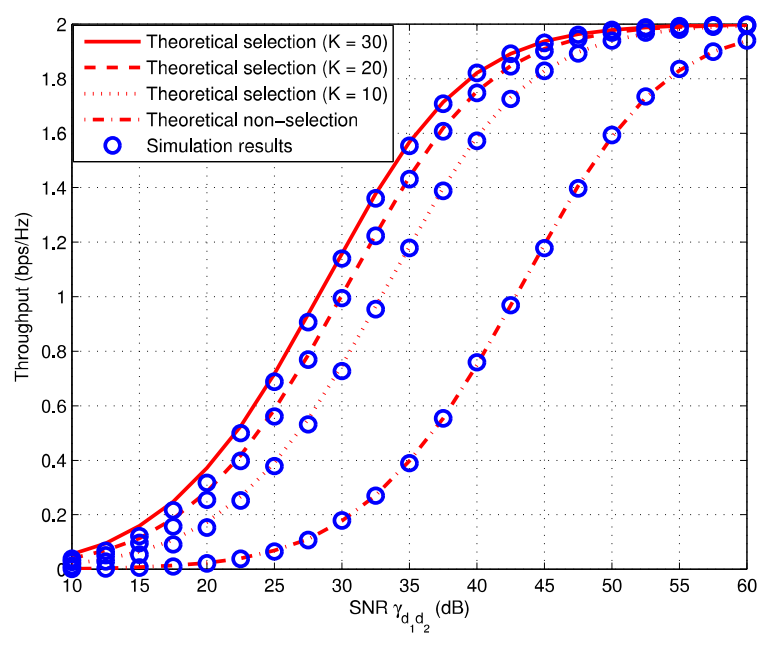

Fig. 3. Theoretical $v s$ numerical throughput of FD-D2D with different number of available users, where $I_{t h}=1, \gamma_{d_{1} b}=15 \mathrm{~dB}, \gamma_{s d_{2}}=30 \mathrm{~dB}, \gamma_{d_{1} d_{1}}=$ $\gamma_{d_{2} d_{2}}=5 \mathrm{~dB}$ and $R_{T}=1$.

Fig. 4 shows throughput $v s$ residual self-interference $\gamma_{d d}=$ $\gamma_{d_{1} d_{1}}=\gamma_{d_{2} d_{2}}$ for half and full duplex D2D protocols, where $R_{T}=1 \mathrm{bps} / \mathrm{Hz}, \gamma_{d_{1} b}=\gamma_{d_{2} b}=10 \mathrm{~dB}$ and $\gamma_{s d_{1}}=\gamma_{s d_{2}}=10$ $\mathrm{dB}$. According to [13], radio transmissions always encounter a bandwidth constraint that limits maximum SI cancellation, therefore, it is useful to consider the different residual SI SNR which can affect the performance of full duplex D2D scheme. In order to compare half and full duplex D2D scenarios fairly, in Fig. 4, we set $I_{t h}=2$ and 1 for half and full duplex D2D, respectively. It is clearly shown that, when residual SI increases, the throughput of FD-D2D is adversely 


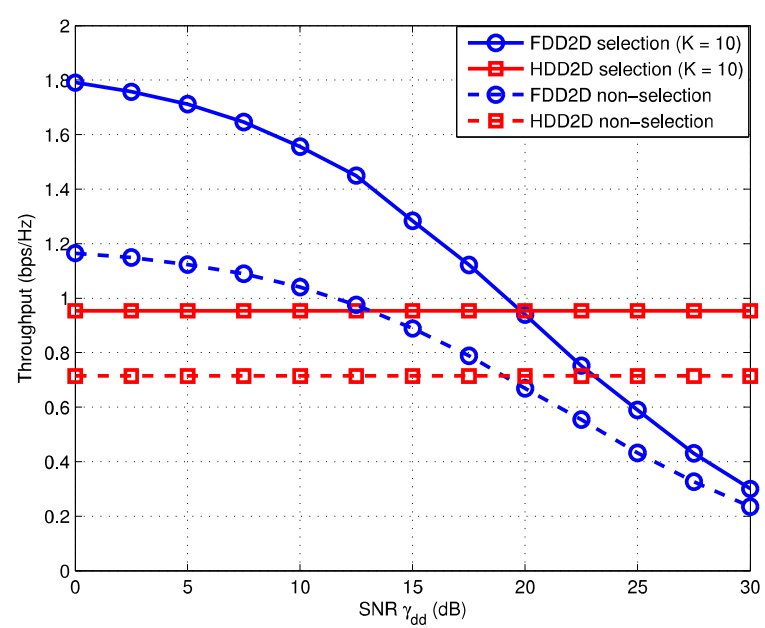

Fig. 4. Throughput $v s$ residual self-interference $\gamma_{d d}$ for half and full duplex D2D protocols with different number of available users, where $R_{T}=1$ $\mathrm{bps} / \mathrm{Hz}, \gamma_{d_{1} b}=\gamma_{d_{2} b}=10 \mathrm{~dB}$ and $\gamma_{s d_{1}}=\gamma_{s d_{2}}=10 \mathrm{~dB}$

affected, but the throughput of HD-D2D keeps a constant. Furthermore, the throughput of HD-D2D with the proposed selection scheme $(K=10)$ or non-selection are always less than that of FD-D2D, when $\gamma_{d d}$ is less that 20 or $19 \mathrm{~dB}$, respectively. Therefore, hybrid-duplex D2D can be selected between half and full duplex according to different residual SI to enhance the throughput of D2D link.

\section{Vi. Conclusion}

This paper studied the best cellular user selection scheme in a hybrid-duplex D2D cellular system. The integral form of the throughput of the hybrid-duplex D2D was derived. The result showed that hybrid-duplex D2D can be selected between half and full duplex according to different residual SI to enhance the throughput of D2D link. We are aware of the fact that practical systems may be more complicated than the system considered in this paper. Nonetheless, the analysis in this paper provides useful insights and a solid basis for further analysis.

\section{ACKNOWLEDGEMENTS}

The research leading to these results has received funding from the European Union Seventh Framework Programme (FP7/2007-2013) under grant agreement NO. 316369 - project DUPLO. We also would like to acknowledge the support of the University of Surrey 5GIC (http://www.surrey.ac.uk/5gic) members for this work.

\section{REFERENCES}

[1] F. Boccardi, R. W. Heath, A. Lozano, T. L. Marzetta, and P. Popovski, "Five disruptive technology directions for 5G," IEEE Commun. Mag., vol. 52, no. 2, pp. 74-80, Feb. 2014.
[2] H. Wang, J. Lee, S. Kim, and D. Hong, "Capacity enhancement of secondary links through spatial diveristy in spectrum sharing," IEEE Trans. Wireless Commun., vol. 9, no. 2, pp. 494-499, Feb. 2010.

[3] K. Doppler, M. Rinne, C. Wijting, C. B. Ribeiro, and K. Hugl, "Deviceto-device communication as an underlay to LTE-Advanced networks," IEEE Commun. Mag., vol. 47, no. 12, pp. 42-49, Feb. 2009.

[4] C. H. Yu, O. Tirkkonen, K. Doppler, and C. Ribeiro, "Power optimization of Device-to-Device communication underlaying cellular communication," in Proc. IEEE International Conference on Communications (ICC), Dresden, Germany, June 2009.

[5] X. Chen, L. Chen, M. Zeng, X. Zhang, and D. Yang, "Downlink resource allocation for Device-to-Device communication underlaying cellular networks," in Proc. IEEE International Symposium on Personal Indoor and Mobile Radio Communications (PIMRC), Sydney, Australia, June 2012.

[6] H. Ju, E. Oh, and D. Hong, "Improving efficiency of resource usage in two-hop full duplex relay systems based on resource sharing and interference cancellation," IEEE Trans. Wireless Commun., vol. 8, no. 8, pp. 3933-3938, Aug. 2009.

[7] T. Riihonen, S. Werner, and R. Wichman, "Optimized gain control for single-frequency relaying with loop interference," IEEE Trans. Wireless Commun., vol. 8, pp. 2801-2806, June 2009.

[8] S. Ali, N. Rajatheva, and M. Latva-aho, "Full-Duplex Device-to-Device communication in cellular networks," in Proc. European conference on Networks and Communications (EuCNC), Bologna, Italy, June 2014.

[9] K. T. Hemachandra, N. Rajatheva, and M. Latva-aho, "Sum-rate analysis for full-Duplex underlay Device-to-Device networks," in Proc. IEEE Wireless Communications and Networking Conference (WCNC), Istanbul, Turkey, April 2014.

[10] A. Ghasemi and E. S. Sousa, "Fundamental limits of spectrum-sharing in fading environment," IEEE Trans. Wireless Commun., vol. 6, no. 2, pp. 649-658, Feb. 2007.

[11] K. Hamdi, W. Zhang, and K. B. Letaief, "Power control in cognitive radio systems based on spectrum sensing side information," IEEE Intl. Conf. Commun., Glasgow, UK, June 2007.

[12] A. Goldsmith, S. A. Jafar, I. Maric, and S. Srinivasa, "Breaking spectrum gridlock with cognative radios: an information theoretic perspective," Proceedings of the IEEE, vol. 97, no. 5, pp. 894-914, May 2009.

[13] M. Jain, J. Choi, T. M. Kim, D. Bharadia, S. Seth, K. Srinivasan, P. Levis, S. Katti, and P. Sinha, "Practical, real-time, full duplex wireless," in Proc. IEEE MobiCom, Las Vegas, Nevada, USA, Sep. 2011. 